\title{
Inner polar ionized-gas disks and properties of their host galaxies
}

\author{
Olga K. Sil'chenko \\ Sternberg Astronomical Institute, Lomonosov Moscow State University, \\ University av. 13, 119991 Moscow, Russia \\ email: olga@sai.msu.su
}

\begin{abstract}
I have analyzed line-of-sight velocity fields of the stellar and ionized-gas components for the volume-limited sample of nearby lenticular galaxies by using the raw data of the ATLAS-3D survey undertaken with the integral-field spectrograph SAURON. Among 200 nearby lenticular galaxies, I distinguish 20 cases of nearly orthogonal rotation of the inner ionized gas with respect to the central stellar components; so I estimate a frequency of the inner polar disks in nearby S0 galaxies as $10 \%$. Properties of the central stellar populations - mean ages, metallicities, magnesium-to-iron ratios - are derived through the Lick indices. The typical stellar population properties of the polar-disk host galaxies are exactly the same as the stellar population properties of the complete sample.
\end{abstract}

Keywords. galaxies: elliptical and lenticular, cD - galaxies: evolution - galaxies: formation

\section{Introduction: Inner polar rings, what is it?}

Everybody knows well such rare but spectacular phenomenon as large-scale polar rings, when the outer gas, and sometimes young stars, rotates in the plane highly inclined to the main galactic disks. However starting from 1990ties, inner analogs of the large-scale polar rings have become to be found. Inner polar rings, or disks, represent circumnuclear, regularly rotating ionized gas which rotation axis is strongly inclined to the rotation axis of the central stellar component - by more than 50 degrees. Recently Moiseev (2012) has collected a list of the inner polar disks found to date and has analyzed their properties. From the statistics of 47 galaxies, it becomes clear that the inner polar disks are indeed polar: even taken over the range of the inclinations to the stellar disks $>50$ degrees, they tend to concentrate to the value of $\Delta i=90^{\circ}$. It is not a surprise because from the dynamical point of view the polar plane is stable against precession. The typical radii of the inner polar disks are $0.2-2 \mathrm{kpc}$, the outer border being real and the inner one being an artifact of the finite spatial resolution of our observations. The inner polar disks are met mostly in early-type disk galaxies, S0s-Sbs, but a few are found in very late-type dwarfs.

A main method by which we can identify inner polar disks is the analysis of twodimensional velocity fields both for the ionized-gas and stellar components, to derive the spatial orientations of their rotation planes by applying tilted-ring approach. It requires the data of 3D spectroscopy. After the Moiseev's review publication, I have undertaken the further search of the inner polar disks by using the rich raw data of the IFU SAURON collected in the frame of the survey ATLAS-3D (Cappellari et al. 2011) and stored in the open ING archive of the CASU Astronomical Data Centre at the Institute of Astronomy, Cambridge. About 150 nearby S0 galaxies of this survey have been analyzed. In this contribution I present the results of my analysis. 

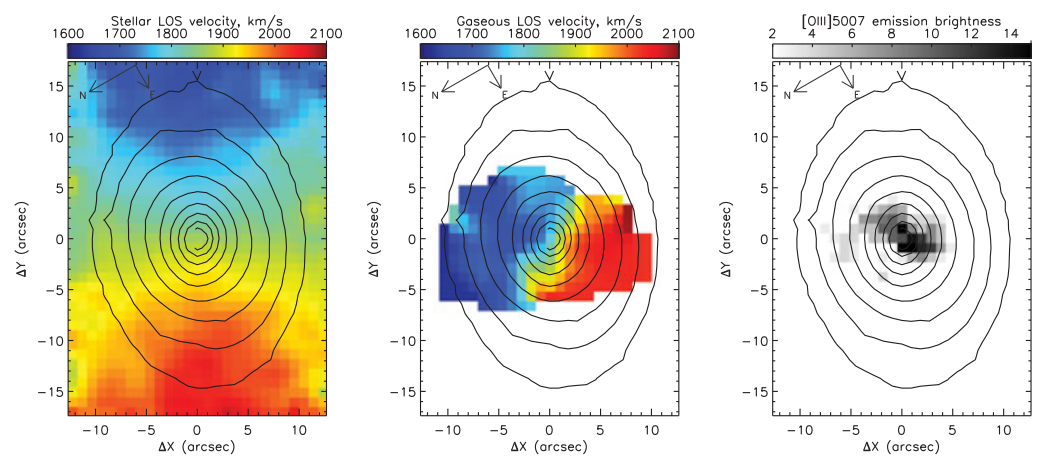

Figure 1. Three maps for NGC 5507 derived from the SAURON data: stellar LOS velocity field (left), ionized-gas LOS velocity field (center), and surface brightness distribution for the gas emission line $[\mathrm{OIII}] \lambda 5007$ (right).

\section{Inner polar disks in the S0s of the ATLAS-3D survey}

By reducing the raw SAURON data for 149 S0 galaxies observed in 2007-2008 in the frame of the ATLAS-3D survey (Cappellari et al. 2011), I have calculated line-of-sight (LOS) velocity fields for the stellar and ionized-gas (when present) components. These 2D LOS-velocity distribution have been analyzed by a tilted-ring approach in a particular modification by Alexei Moiseev (Moiseev et al. 2004) allowing to estimate parameters of the spatial orientation of the rotation planes for both components, inclinations $i_{s, g}$ and position angles of the lines of nodes, $P A_{s, g}$. After that, the mutual inclinations of the stellar and gaseous rotation planes have been calculated by using the following formula (Moiseev 2012):

$$
\cos \Delta i= \pm \cos \left(P A_{s}-P A_{g}\right) \sin i_{s} \sin i_{g}+\cos i_{s} \cos i_{g} .
$$

The equation has two solutions because typically we do not know what side of a galactic disk is closer to us. By making these calculations, among 149 S0s of the ATLAS-3D survey, I have found 6 new cases of the nearly polar inner ionized-gas disks with $\Delta i>50^{\circ}$. These are NGC $2962(\Delta i=50$ or $114 \mathrm{deg})$, NGC $3499(\Delta i=61$ or $68 \mathrm{deg})$, NGC $3648(\Delta i=68$ or $88 \mathrm{deg}$ ), NGC 4690 ( $\Delta i=64$ or $68 \mathrm{deg}$ ), NGC 1121 and NGC 5507 (edge-on?). As an illustration of the data quality, for the latter galaxy, NGC 5507, I give here three maps (Fig. 1): stellar LOS velocity field, ionized-gas LOS velocity field, and surface brightness of the gas emission line [OIII] $\lambda 5007$ which demonstrates clearly that we deal with the gaseous disk edge-on, in full agreement with the gas kinematical major axis orientation. Also the inner polar rings have been noted in the isolated S0 UGC 9519 through the central dust distribution though the ionized-gas velocity field is rather irregular in this galaxy (Katkov et al. 2014), and in NGC 4684 by Davis et al. (2011) who found the kinematical major axes stars/ionized-gas misalignment of about 90 deg in this edge-on galaxy. Taking these 8 galaxies together with those listed earlier by Moiseev (2012), and considering the full ATLAS-3D sample including the galaxies observed earlier than 2007, we obtain 20 inner polar ionized-gas disks in the sample of 200 nearby S0 galaxies. Since the sample of the early-type galaxies in the ATLAS-3D survey is complete within the volume limited by $D<42 \mathrm{Mpc}$ and above the luminosity $M_{K}<-21.5$ (Cappellari et al. 2011), I conclude that the frequency of the inner polar disks among the nearby S0 galaxies is about $10 \%$. So the phenomenon of the inner polar disks is much more common than the large-scale polar rings (Moiseev et al. 2011). 

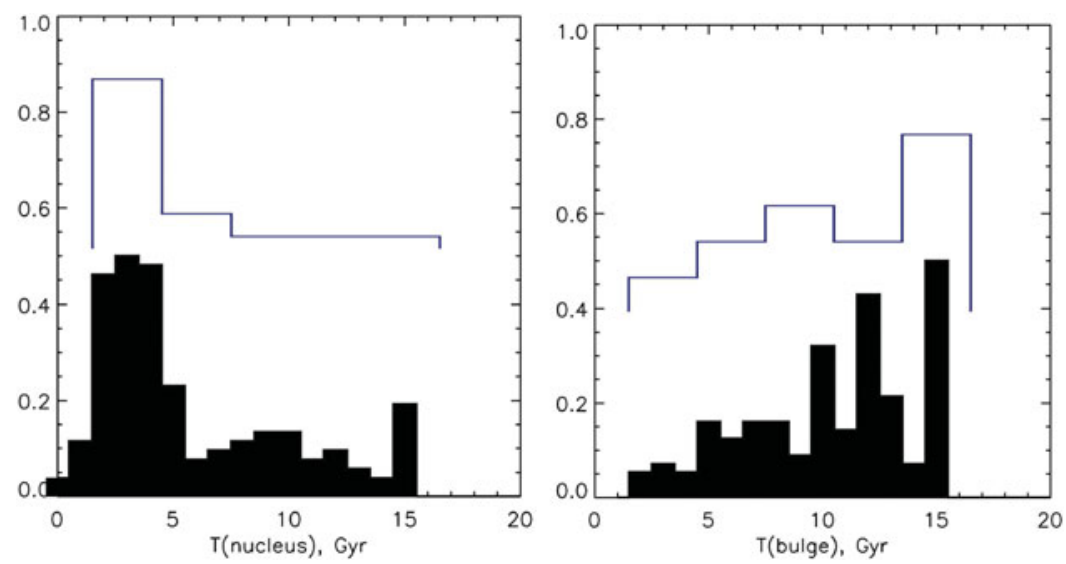

Figure 2. The distributions of the SSP-equivalent ages for the nuclei (left) and bulges (right) of the ATLAS-3D S0s: filled histograms present the total sample, while the blue line - the inner polar-disk hosts.

\section{So hosts of the inner polar disks: are they special?}

I have also analyzed properties of the stellar populations in the nuclei and the bulges of 149 S0 galaxies of the ATLAS-3D survey. To get them, I have calculated Lick indices (Worthey et al. 1994) $\mathrm{H} \beta, \mathrm{Mgb}$, and Fe5270 in every spaxel for every galaxy, then I have averaged them for the nuclei, $R<1^{\prime \prime}$, and for the bulges, taken in the rings of $4^{\prime \prime}<R<7^{\prime \prime}$ for galaxies close to face-on and along the minor axes in $4^{\prime \prime}-6^{\prime \prime}$ from the centers for galaxies close to edge-on. I have confronted the obtained mean indices to the Simple Stellar Populations (SSP) models by Thomas et al. (2003) which allowed to take into account different abundance ratios $\mathrm{Mg} / \mathrm{Fe}$ and to obtain SSP-equivalent (luminosity-averaged) ages and metallicities. With these data in hands, I have constructed the distributions of the ages and metallicities, both for the nuclei and the bulges. I wanted to search for particular ecolutionary paths of the hosts of the inner polar disks. The comparison of the distributions for the total sample with those for the hosts of the inner polar disks is presented in Fig. 2 and Fig. 3. Curiously, the polar-disk host galaxies do not differ in any sense from the other lenticular galaxies. Even a rather exotic bimodal age distribution of the stellar nuclei where the majority is young, of 2-5 Gyr old, but a significant fraction of very old stellar nuclei is also present, is perfectly reproduced by the inner polar-disk hosts.

Further on, the hosts of the inner polar disks fill up the full range of galaxy masses which was probed by the ATLAS-3D survey, and take part in all known correlations. Interestingly, the well-known correlation of the stellar population ages with the stellar velocity dispersion for the nuclei holds only for the rather young nuclei while the old nuclei are homogeneously distributed among the galaxies of various masses.

\section{Conclusions}

By analyzing the kinematical maps for the full lenticular-galaxy sample of the ATLAS3D survey, I have found a few new cases of the nearly polar rotation of the circumnuclear ionized gas and have estimated the frequency of the inner polar ionized-gas disks as $10 \%$. Inner polar disks can be met in lenticular galaxies of any mass and over the environments of any density, including some cases in completely isolated galaxies. The properties of the stellar populations of the nuclei and circumnuclear bulges derived through the Lick index 

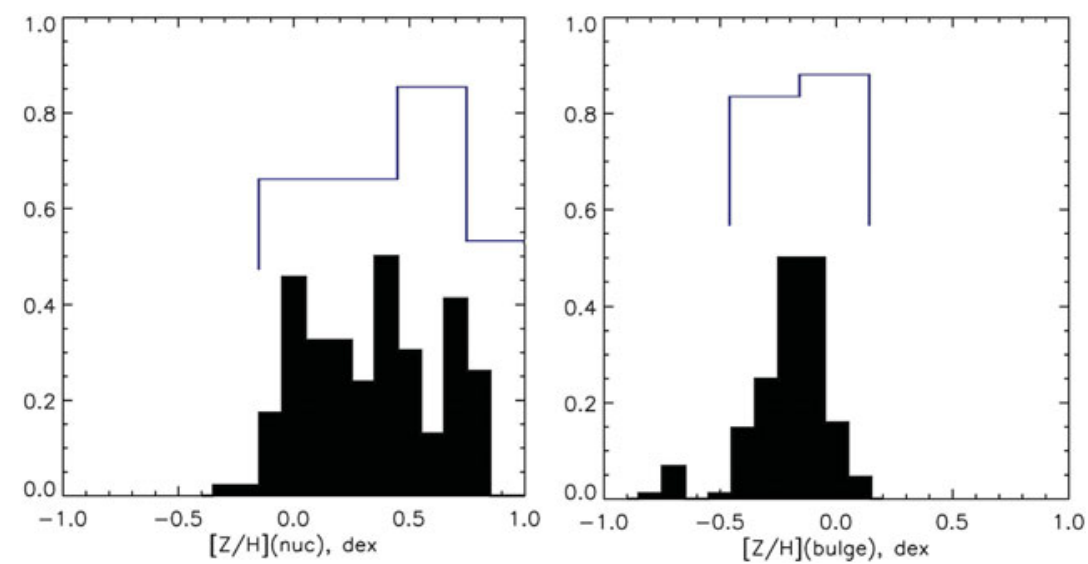

Figure 3. The distributions of the stellar metallicities for the nuclei (left) and bulges (right) of the ATLAS-3D S0s: filled histograms present the total sample, while the blue line - the inner polar-disk hosts.

modelling have appeared to be the same in the host galaxies of the inner polar disks and over the full sample, including a large fraction of young stellar nuclei, of $2-5$ Gyr old.

\section{Acknowledgements}

I acknowledge financial support from the IAU to attend this Symposium. The present contribution makes use of data obtained from the Isaac Newton Group Archive which is maintained as part of the CASU Astronomical Data Centre at the Institute of Astronomy, Cambridge.

\section{References}

Cappellari, M., Emsellem, E., Krajnovic, D., McDermid, R. M., Scott, N., et al. 2011, MNRAS, 413,813

Davis, T. A., Alatalo, K., Sarzi, M., Bureau, M., Young, L. M., et al. 2011, MNRAS, 417, 882

Katkov, I. Yu., Sil'chenko, O. K., \& Afanasiev, V. L. 2014, MNRAS, 438, 2798

Moiseev, A. V., Valdés, J. R., \& Chavushyan, V. H. 2004, A\& A, 421, 433

Moiseev, A. V., Smirnova, K. I., Smirnova, A. A., \& Reshetnikov, V. P. 2011, MNRAS, 418, 244

Moiseev, A. V. 2012, Astrophys. Bull., 67, 147

Thomas, D., Maraston, C., \& Bender, R. 2003, MNRAS, 339, 897

Worthey, G., Faber, S. M., González, J. J., \& Burstein, D. 1994, ApJS, 94, 687 